\title{
A patient-centered approach: treatment of obsessive compulsive disorder
}

\section{Introduction}

Obsessive Compulsive Disorder is a serious mental health disorder that causes a patient significant distress, yet it is often challenging both for the patient to access treatment, and for the provider to determine the best treatment modality. This article outlines a guide to treatment of OCD in an outpatient population. Obsessive compulsive disorder is characterized by intrusive distressing thoughts called obsessions and actions intended to prevent harm and alleviate anxiety called compulsions. ${ }^{1}$ While everyone occasionally wonders whether they remembered to lock the door, these thought are so persistent and distressing to the OCD patient that he or she must repeatedly check to ensure that it is locked. Likewise, no one wants to catch influenza, but a patient with OCD may repeatedly wash his or her hands and anything else that may have become contaminated with the virus. ${ }^{2}$ Moreover, if prevented from performing these tasks, a patient with OCD is plagued with anxiety. These behaviors can have a significant impact on the OCD patient's life, but not every OCD-sufferer requires the same level of treatment in order to achieve recovery. Therefore, a stepped approach is necessary to give each patient the intensity of treatment he or she requires and no more, starting with bibliotherapy combined with counseling, moving to a trial of fluoxetine if that is ineffective, and then continuing on to ICBT for non-responders, or therapist directed exposure response prevention for those patients who may benefit from more direct contact with the therapist. Bibliotherapy combined with bi-weekly counseling is a low-key, non-intrusive first step in the treatment of obsessive compulsive disorder. The patient is given a self-help book about OCD, along with a reading schedule, and has a brief meeting every other week with his or her therapist to get questions answered and examine sources of stress. ${ }^{3}$ The book explains exposure and response prevention and helps patients to begin using this treatment technique. ${ }^{3}$ For some patients, the book and therapist check-ins provide enough guidance to successfully eliminate the bothersome OCD symptoms and live a more productive, less anxietydriven life. While it may require some motivation to change, patients impaired enough by their symptoms to seek treatment are likely adequately impaired to have sufficient motivation to change.

Patients who have not seen adequate improvement at the end of six weeks of bibliotherapy and counseling may want to consider a trial of fluoxetine. If no improvement has been seen with the bibliotherapy and counseling, the patient may discontinue that treatment modality during the fluoxetine trial; however, if the patient saw some improvement, but was not satisfied with the extent of the improvement, he or she may benefit from continuing to meet with the therapist while on the medication. Some patients may be too depressed to find the motivation to work hard and benefit fully from the previous step. As an antidepressant, fluoxetine may address that condition in addition to directly influencing the anxiety behind the obsessive thoughts and compulsive actions. Because fluoxetine is less effective for patients with checking compulsions, these patients may choose to skip this step; for other OCD patients, this treatment modality may be sufficient to alleviate their symptoms.
Volume 3 Issue I - 2015

\section{Victoria H Anderson}

St. Louis College of Pharmacy, USA

Correspondence: Victoria H.Anderson, St. Louis College of
Pharmacy, USA, Tel 65।2534899.

Email victoria.anderson@stlcop.edu

Received: February 21, 2015 | Published: December 04, 2015

After eight weeks on fluoxetine, some patients may still not have seen an adequate response to treatment. For these patients, more intensive therapy may be necessary to gain control over their OCD. Depending on whether or not they saw any gain from the fluoxetine, the patients may again choose whether to continue taking fluoxetine while participating in therapy or to discontinue the medication. For these patients who have failed to have an adequate response to either the bibliotherapy and bi-weekly counseling or the fluoxetine, and for those who were not successful with the bibliotherapy and counseling but skipped the fluoxetine trial, internet CBT may be a useful option. With internet CBT, the patient is granted access to successive online modules as the therapist deems he or she is ready, and is provided feedback on assignments. ${ }^{2}$ These modules focus on helping the patient prepare for and execute ERP exercises, with a therapist available within twenty-four hours for questions and support. ${ }^{2}$ In a study conducted in Sweden, internet-based CBT was found to be as effective as traditional CBT, but for patients with lower levels of computer literacy or who may prefer more direct contact with a therapist twice a week, exposure and response prevention with a therapist may be a useful alternative. ${ }^{2}$ This more intensive therapy should benefit those who did not see the results they desired from the less intense levels of treatment. Starting with bibliotherapy and counseling, moving to fluoxetine if necessary, and saving intensive exposure and response prevention therapy for those who do not respond to treatments of lesser intensity allows for a stepped approach to treatment, to give each patient the level of treatment they need without giving them more than necessary. This empirically proven approach makes living a more normal life a much more accessible goal for patients because they know they will not immediately enter into a time-consuming, stressful, highintensity treatment when seeking help. By only giving the patient the level of treatment he or she needs, it also makes treatment more affordable, and therefore more approachable. Additionally, because the lower levels and ICBT do not require a large amount of time with trained therapists, therapists will have more time available for those patients who really require their services to recover from OCD. This will maximize the number of people able to move from a distressing amount of worry to a more normal adaptive level, enabling them to live a more fulfilling life. 


\section{Acknowledgements}

None.

\section{Conflict of interest}

Author declares that there is no conflict of interest.

\section{References}

1. Farnam A, Goreishizadeh M, Farhang S. Effectiveness of fluoxetine on various subtypes of obsessive-compulsive disorder. Arch Iranian Med. 2008;11(5):522-525.
2. Andersson E, Enander J, Andren P, et al. Internet-based cognitive behaviour therapy for obsessive-compulsive disorder: A randomized controlled trial. Psychological Medicine. 2012;42(10):2193-2203.

3. Tolin DF, Diefenbach GJ, Gilliam CM. Stepped care versus standard cognitive-behavioral therapy for obsessive-compulsive disorder: A preliminary study of efficacy and costs. Depression and Anxiety. $2011 ; 28(4): 314-323$ 